\title{
The relationship between fragility, configurational entropy and the potential energy landscape of glass forming liquids
}

\author{
Srikanth Sastry \\ Jawaharlal Nehru Centre for Advanced Scientific Research,Jakkur Campus, Bangalore 560064, INDIA
}

Glass is a microscopically disordered, solid form of matter that results when a fluid is cooled or compressed in such a fashion that it does not crystallise. Almost all types of materials are capable of glass formation - polymers, metal alloys, and molten salts, to name a few. Given such diversity, organising principles which systematise data concerning glass formation are invaluable. One such principle is the classification of glass formers according to their fragility [i]]. Fragility measures the rapidity with which a liquid's properties such as viscosity change as the glassy state is approached. Although the relationship between features of the energy landscape of a glass former, its configurational entropy and fragility have been analysed previously (e. g., [2] ), an understanding of the origins of fragility in these features is far from being well established. Results for a model liquid, whose fragility depends on its bulk density, are presented in this letter. Analysis of the relationship between fragility and quantitative measures of the energy landscape (the complicated dependence of energy on configuration) reveal that the fragility depends on changes in the vibrational properties of individual energy basins, in addition to the total number of such basins present, and their spread in energy. A thermodynamic expression for fragility is derived, which is in quantitative agreement with kinetic fragilities obtained from the liquid's diffusivity.

Glass forming liquids grow increasingly viscous upon cooling, till the viscosity becomes so large that they fail to flow on experimental time scales. While remaining microscopically disordered like a liquid, they manifest mechanical properties of a solid; $i$. e., they transform to a glass. By convention, the glass transition temperature $T_{g}$ is that where the viscosity reaches a value of $10^{12} \mathrm{~Pa}$ sec. The approach to this large viscosity, however, differs from one liquid to another. When displayed in an Arrhenius plot [ $\log ($ viscosity) vs. inverse temperature $1 / T]$, some liquids $(e . g$., silica) show a steady, linear increase, while others display a much steeper dependence on $1 / T$ [1].3], as illustrated in Fig. 1(a) (inset). The former are strong liquids, and the latter, fragile. This range of behaviours is implicit in the Vogel-Fulcher-Tammann-Hesse (VFT) form, observed to describe the $T$ dependence of viscosity (as well as diffusivity and relaxation times) in many glass formers. The VFT relation may be written as

$$
\eta=\eta_{0} \exp \left[\frac{1}{K_{V F T}\left(T / T_{0}-1\right)}\right],
$$

where $T_{0}$ is the temperature of apparent divergence of viscosity, and $K_{V F T}$ is a material specific parameter quantifying the kinetic fragility; more fragile liquids have larger $K_{V F T}$ values. This behaviour of viscosity correlates with the jump in heat capacity at the glass transition - more fragile the liquid, sharper and bigger the jump. Rationalisation of this correlation comes from the Adam-Gibbs relationship [- [- which predicts a dependence of viscosity on the configurational entropy $S_{c}$ (described later) of the liquid:

$$
\eta=\eta_{0} \exp \left[\frac{A}{T S_{c}}\right],
$$

which results in the VFT relation if $S_{c}$ has the form $T S_{c}=K_{A G}\left(T / T_{K}-1\right)$ [20], where $T_{K}$ is the ideal glass transition or Kauzmann temperature [4] below which $S_{c}=0$. The jump in heat capacity is proportional to $K_{A G}$, and is larger for more fragile glass formers.

Fragility is analysed here for a binary mixture of particles interacting via the Lennard-Jones potential, widely studied as a model glass former [5 9 . The $T$ dependence of diffusivity $D$ (to characterise dynamics) and configurational entropy are obtained for a range of bulk densities $\rho$. The diffusivities $D(\rho, T)$, are shown in Fig. 1(a) in a 'fragility plot': $-\log [D(\rho, T)]$ vs. $T_{r} / T$, where $T_{r}$ is a reference temperature (akin to $T_{g}$ in the usual fragility plot) at which $D$ at each density reaches a fixed, small value. By comparison with the inset, the liquid is seen to become more fragile as its density increases. Fig. 1(b) shows the kinetic fragility index, $K_{V F T}$, obtained from VFT (Eq.(1)) fits to diffusivity data at each density. The density dependence of $K_{V F T}$ quantifies the increase of fragility with density, apparent from data in Fig. 1(a).

A thermodynamic explanation of fragility, as discussed above (also [10,11]), lies in explaining the rapidity of change with $T$ of the configurational entropy, $S_{c}$. In the inherent structure (IS) formalism [12 13] employed here, the configuration space of a liquid is divided into basins of local potential energy minima ('inherent structures'). $S_{c}$ at a 
given $T$ derives from the multiplicity of local potential energy minima sampled by the liquid at that $T$, and equals the difference of the total entropy and the vibrational entropy of typical basins sampled, $S_{v i b}$ :

$$
S_{c}(\rho, T)=S_{\text {total }}(\rho, T)-S_{v i b}(\rho, T) .
$$

For all the results presented here, the basin entropy $S_{v i b}$ is calculated by approximating each basin as a harmonic well (valid at sufficiently low $T$ ) 7 7 9,14. In this approximation, the entropy of a given basin, arising from vibrational motion within the basin (the suffix vib emphasizes this point) is given by

$$
S_{v i b}=k_{B} \sum_{i=1}^{3 N} 1-\log \left(\frac{h \nu_{i}}{k_{B} T}\right),
$$

where $\nu_{i}$ are the vibrational frequencies of the given basin, $k_{B}$ is Boltzmann's constant and $h$ is Plank's constant. The basin free energy is $F_{v i b}=U_{v i b}-T S_{v i b}$, where $U_{v i b}=3 N k_{B} T$ is the internal energy. Apart from the explicit temperature dependence of $S_{v i b}$ above, an implicit dependence also exists because vibrational frequencies change from one basin to another, and, different basins are sampled at different temperatures. From the expression above, it is clear that the entropy difference between any two basins arises solely from the difference in their vibrational frequencies, and is independent of temperature.

The configurational entropy is evaluated in this letter using two methods, referred to as the thermodynamic integration (TI) method, and the potential energy landscape (PEL) method, respectively. In both cases, inherent structures are obtained by performing local potential energy minimisation for a subset of configurations sampled by a liquid at each $\rho$ and $T$. Vibrational frequencies are calculated for each inherent structure, and the basin entropy $S_{v i b}$ is obtained from Eq. (4). In the TI method (described in detail elsewhere [7] 9]; see also caption of Fig. 2), the total entropy $S_{\text {total }}$ is obtained using pressure and internal energy values from simulations. Eq. (3) is used to obtain $S_{c}$. In contrast, the PEL method is based on constructing the distribution of inherent structures in order to calculate $S_{\text {total }}$ and $S_{c}$, as described later.

Fig. 2(a) shows the Adam-Gibbs plot, where $\log D$ is plotted against $\left(T S_{c}\right)^{-1}$. The Adam-Gibbs relation (Eq. (2)) is seen to apply well at each density, as also observed previously for the hard sphere liquid [15] and water [16]. Fig. 2(b) shows (as lines) the $T$ dependence of $T S_{c}$ (from TI). All curves are nearly linear, but with slight increases of slope at lower $T$. The slopes of $T S_{c}$ (in the $T$ range where diffusivities are measured), $K_{A G}^{T I}$, are used as the quantitative index of thermodynamic fragility, and are plotted in Fig. 1(b). $K_{A G}^{T I}$ are nearly the same as the kinetic fragility index $K_{V F T}$, thereby validating the idea that the rate of increase of $T S_{c}$ does indeed determine the fragility.

However, this analysis provides no insight into how the fragility relates to features of the energy landscape. To this end, the distribution of energy minima, and properties of individual basins, are now examined, at fixed density $\rho$. The probability distribution $P(\Phi, T)$ that an inherent structure of energy $\Phi$ is sampled at temperature $T$ depends on (a) the energy $\Phi,(\mathrm{b})$ the structure of the basin, contained in the basin free energy $F_{v i b}(\Phi, T)$ and (c) the number density of inherent structures with energy $\Phi, \Omega(\Phi)$ [12, 13, 1, 14, 17]:

$$
P(\Phi, T)=\Omega(\Phi) \exp \left[-\beta\left(\Phi+F_{v i b}(\Phi, T)\right)\right] / Q_{N}(\rho, T),
$$

where $Q_{N}$, the partition function, normalises $P$ and is given by $Q_{N}=\int d \Phi P(\Phi, T)$ over all possible $\Phi$. The configurational entropy density is defined by $\mathcal{S}_{c}(\Phi) \equiv k_{B} \ln \Omega(\Phi)$ and is related to the $T$ dependent configurational entropy by $S_{c}(T)=\int d \Phi \mathcal{S}_{c}(\Phi) P(\Phi, T)$. The quantities $P(\Phi, T), F_{v i b}$ and $Q_{N}$ are obtained from simulations [7, 14, 9, 17], and are used to invert the relation in Eq. (5) to obtain the configurational entropy density $\mathcal{S}_{c}(\Phi)$. The same $\mathcal{S}_{c}(\Phi)$ estimates must result (though not for the same range of $\Phi$ ) for different $T$, when the harmonic assumption is valid, which is indeed seen to be the case from data shown in Fig. 3(a). With increasing density, the maximum $\mathcal{S}_{c}(\Phi)$ value reached decreases, and the distribution becomes broader. It has been argued [18.19] that the distribution $\Omega(\Phi)$ should be Gaussian (equivalently, $\mathcal{S}_{c}(\Phi)$ an inverted parabola). Data in Fig. 3(a) are fully consistent with this expectation, and are fitted to the form,

$$
\frac{\mathcal{S}_{c}(\Phi)}{N k_{B}}=\alpha-\frac{\left(\Phi-\Phi_{o}\right)^{2}}{\sigma^{2}}
$$

where $\alpha$ is the height of the parabola and determines the total number of configurational states, $i$. $e$. energy minima (the total number is proportional to $\exp (\alpha N)), \Phi_{0}$ and $\sigma^{2}$ respectively define the mean and the variance of the distribution. The fits are displayed in Fig. 3(a) and the fit parameters listed in the figure caption. Speedy [2] has considered a model of the thermodynamics of a glass former with a Gaussian distribution of configurational states, 
and the basin specific heat that does not depend on the internal parameter (here the IS energy). He predicts that the fragility of a liquid should be proportional to the logarithm of the number of states, $i$. e e. on $\alpha$. From Fig. 3(a) it is clear that $\alpha$ values, and hence the number of states, decrease with increasing density $\rho$. But the fragilities (Fig. 1(b)) show the opposite trend. However, fragility has an additional dependence (implicit in ref. [2]) on the variance of the distribution $\sigma$ (see equation (6)).

Further, the variation of vibration frequencies, and hence the basin entropy, from basin to basin, also contributes to the fragility of the liquid. Fig. 3(b) displays the $T$ independent (see above) basin entropy difference at a given energy $\Phi$ with respect to the value at $\Phi_{0}, \Delta S_{v i b}(\Phi) \equiv S_{v i b}(\Phi, T)-S_{v i b}\left(\Phi_{0}, T\right)$. At all densities, $\Delta S_{v i b}(\Phi)$ displays a clear $\Phi$ dependence, which is inconsistent with assumptions in [2]. Although such basin dependence of vibrational frequencies and hence the basin entropy have been discussed previously [21 30], with reference to both experimental data and model calculations, its implications on a liquid's fragility have not clearly been established. Fig. 3(b) shows that basins at higher energy (which are occupied at higher temperature) have lower basin entropies, or, higher frequencies (see Eq. (四)). This trend, contrary to what one expects for systems studied experimentally, arises because the constant density model system here cannot expand when $T$ is raised.

The dependence of $\Delta S_{v i b}(\Phi)$ on $\Phi$ is to a very good extent linear. Hence, for each density, $\Delta S_{v i b}$ is fit to the form $\Delta S_{v i b}(\Phi)=\delta S\left(\Phi-\Phi_{0}\right)$ with fit parameters listed in the caption of Fig. 3. The basin free energy, which can be written as $F_{v i b}(\Phi, T)=F_{v i b}\left(\Phi_{0}, T\right)-T \delta S\left(\Phi-\Phi_{0}\right)$, is also linear in $\Phi$. With a Gaussian form for $\Omega$, the partition function $Q_{N}=\int d \Phi P(\Phi, T)(P$ as in Eq. (5) ) is easily evaluated, and results in the following predictions for the $T$ dependence of the average IS energy, and the configurational entropy:

$$
<\Phi>(T)=\Phi_{0}^{e f f}-\frac{\sigma^{2}}{2 N k_{B} T},
$$

where $\Phi_{0}^{e f f}=\Phi_{0}+\frac{\sigma^{2} \delta S}{2 N k_{B}}$, and

$$
T S_{c}(T)=K_{A G}^{P E L}(T) \quad\left(T / T_{K}-1\right) ; \quad K_{A G}^{P E L}(T)=\left(\frac{\sigma \sqrt{\alpha}}{2}+\frac{\sigma^{2} \delta S}{4 N k_{B}}\right)\left(1+\frac{T_{K}}{T}\right)-\frac{\sigma^{2} \delta S}{2 N k_{B}},
$$

where $T_{K}=\sigma\left(2 N k_{B} \sqrt{\alpha}+\sigma \delta S\right)^{-1}$ is the ideal glass transition temperature where $S_{c}\left(T_{K}\right)=0$.

The form above for $S_{c}$ results in the VFT relation, if $K_{A G}^{P E L}(T)$ is constant. This is not quite the case, but the $1 / T$ term becomes rapidly irrelevant for $T>T_{K}$ and $K_{A G}^{P E L}(T)$ approaches a constant. Further, $T S_{c}$ values plotted (as points) in Fig. 2(b) are in extremely good agreement with the ones from thermodynamic integration, including deviations from linearity for small $T / T_{K}$. A check of the overall consistency of the model described here is made in Fig. 3(c), which shows a scaled plot of $\langle\Phi\rangle(T)-\Phi_{0}^{e f f}$ vs. $\frac{\sigma^{2}}{2 N k_{B} T}$. From Eq. (7), one expects $\Phi(T)$ data for all densities to collapse onto a straight line of (negative) unit slope, with expected deviations at high $T$ due to basin anharmonicity [14. Data in Fig. 3(c) clearly meet this expectation, although with noticeable deviations for $\rho=1.35 \rho_{0}$, as also in Fig. 2(b).

The expression for $K_{A G}^{P E L}$ in Eq. (8), the thermodynamic fragility index obtained from energy landscape parameters, shows that fragility depends not only on the multiplicity of states $(\alpha)$ but also on their spread $(\sigma)$, and how much the basin entropy changes from the lowest to the highest energy basins sampled by the liquid. The extent of this change is quantified by parameters $\sigma$ and $\delta S$ (listed in the caption of Fig. 3). Fig. 1(b) shows the average slopes of $T S_{c}$ (from Eq. (8) above, in the $T$ range where diffusivities are measured), $K_{A G}^{P E L}$, which are nearly the same as $K_{A G}^{T I}$, and in very good agreement with the kinetic fragility index $K_{V F T}$. This agreement establishes the quantitative relationship between fragility and the energy landscape of the liquid since $K_{A G}^{P E L}$ is obtained solely in terms of a quantitative description of the liquid's energy landscape.

[1] Angell, C. A. Relaxation in Liquids, Polymers and Plastic Crystals - Strong/Fragile Patterns and Problems. J. Non-Cryst. Solids 131-133, 13-31 (1991).

[2] Speedy, R. J. Relations between a Liquid and Its Glasses. J. Phys. Chem. B 103, 4060-4065 (1999).

[3] Laughlin, W. T. and Uhlmann, D. R. Viscous Flow in Simple Organic Liquids. J. Phys. Chem. 76, 2317-2325 (1972).

[4] Adam, G. and Gibbs, J. H On the Temperature Dependence of Cooperative Relaxation Properties in Glass-Forming Liquids. J. Chem. Phys. 43, 139-146 (1965). 
[5] Kob, W. and Andersen, H. C. Testing mode-coupling theory for a supercooled binary Lennard-Jones mixture: The van Hove correlation function. Phys. Rev. E 51, 4626-4641 (1995).

[6] Sastry, S., Debenedetti, P. G. and Stillinger, F. H. Signatures of Distinct Dynamical Regimes in the Energy Landscape of a Glass Forming Liquid. Nature 393, 554-557 (1998).

[7] Sciortino, F., Kob, W. and Tartaglia, P. Inherent Structure Entropy of Supercooled Liquids. Phys. Rev. Lett. 83, 3214-3217 (1999).

[8] Coluzzi, B., Parisi G. and Verrocchio, P. Lennard-Jones binary mixture: a thermodynamical approach to glass transition. J. Chem. Phys. 112 2933-2944 (2000).

[9] Sastry, S. Liquid Limits: The Glass Transition and Liquid-Gas Spinodal Boundaries of Metastable Liquids. Phys. Rev. Lett. 85 590-5593 (2000).

[10] Ito, K., Moynihan, C. T. and Angell, C. A. Thermodynamic determination of fragility in liquids and a fragile-to-strong liquid transition in water. Nature 398, 492-495 (1999).

[11] Xia, X. and Wolynes, P. G. Fragilities of Liquids Predicted from the Random First Order Transition Theory of Glasses. Proc. Nat. Acad. Sci. 97 2990-2994 (2000).

[12] Stillinger, F. H. and Weber, T. A. Packing Structures and Transitions in Liquids and Solids. Science 225, 983-989 (1984).

[13] Stillinger, F. H. A Topographic View of Supercooled Liquids and Glass Formation. Science 267, 1935-1939 (1995).

[14] Buechner, S. and Heuer, A. The potential energy landscape of a model glass former: thermodynamics, anharmonicities, and finite size effects. Phys. Rev. E 60, 6507-6518 (1999).

[15] Speedy, R. J. The hard sphere glass transition. Mol. Phys. 95 169-178 (1998).

[16] Scala, A., Starr, F. W., La Nave, E., Sciortino, F. and Stanley, H. E. Configurational Entropy and Diffusivity of Supercooled Water. Nature 406 166-169 (2000).

[17] Sastry, S. Evaluation of configurational entropy of a model liquid from computer simulations. Proceedings of Unifying Concepts in Glass Physics, Trieste, 1999, J. Phys. Cond. Mat. 12, 6515-6524 (2000).

[18] Speedy, R. J. and Debenedetti, P. G. The distribution of tetravalent network glasses Mol. Phys. 88 1293-1316 (1996).

[19] Heuer, A. and Buechner, S. Why is the density of inherent structures of a Lennard-Jones type system gaussian? Proceedings of Unifying Concepts in Glass Physics, Trieste, 1999, J. Phys. Cond. Mat. 12, 6535-6543 (2000).

[20] Angell, C. A. Entropy and Fragility in Supercooled Liquids. J. Res. NIST 102, 171-185 (1997).

[21] Goldstein, M. Viscous liquids and the glass transition. V. Sources of the excess specific heat of the liquid. J. Chem. Phys. bf 64, 4767-4774 (1976).

[22] Angell, C. A. and Rao, K. J. Configurational Excitations in Condensed Matter and the Bond Lattice Model for the Liquid-Glass Transition. J. Chem. Phys. bf 57, 470-481 (1972).

[23] Angell, C. A. Ten questions on glassformers, and a real space "excitations" model with some answers on fragility and phase transitions. Proceedings of Unifying Concepts in Glass Physics, Trieste, 1999, J. Phys. Cond. Mat.12, 6463-6476 (2000).

[24] Green, J. L., Ito, K., Xu, K. and Angell, C. A. Fragility in Liquids and Polymers: New, Simple Quantifications and Interpretations. J. Phys. Chem. B 103, 3991-3996 (1999).

[25] Johari, G. P. A resolution for the enigma of a liquid's configurational entropy-molecular kinetics relation. J. Chem. Phys. $1128958-8969$ (2000)

[26] Johari, G. P. Contributions to the entropy of a glass and liquid, and the dielectric relaxation time. J. Chem. Phys. 112 $7518-7523(2000)$.

[27] Yamamuro, O., Tsukushi, I., Lindqvist, A., Takahara, S., Ishikawa, M. and Matsuo, T. Calorimetric Study of Glassy and Liquid Toluene and Ethylbenzene: Thermodynamic Approach to Spatial Heterogeneity in Glass-Forming Molecular Liquids. J. Phys. Chem. B 102 1605-1609 (1998).

[28] Ngai, K. L. and Yamamuro, O. Thermodynamic fragility and kinetic fragility in supercooled liquids: A missing link in molecular liquids. J. Chem. Phys. 111 10403-10406 (1999).

[29] Sciortino, F. and Tartaglia, P. "Extension of the Fluctuation-Dissipation theorem to the physical aging of a model glassforming liquid" Phys. Rev. Lett. (in press).

[30] Starr, F. W., Sastry, S., La Nave, E., Scala, A., Sciortino, F. and Stanley, H. E. "Thermodynamic and structural aspects of the potential energy surface of simulated water" http://arXiv.org/abs/cond-mat/0007487.

Acknowledgements: I thank C. A. Angell, G. P. Johari, K. J. Rao, F. Sciortino, R. Seshadri, R. J. Speedy and U. $\mathrm{V}$. Waghmare for very useful discussions and/or comments on the manuscript.

Correspondence should be addressed to the authour (email: sastry@jncasr.ac.in). 
Figure 1: Fragility Plot of diffusivities, kinetic and thermodynamic fragility indices. Results shown are obtained from molecular dynamics simulations of $204 A$ type and $52 B$ type particles interacting via the Lennard-Jones (LJ) potential. Argon units are used for $A$ type particles: LJ parameter $\epsilon_{A A}=k_{B} \times 119.8 K, \sigma_{A A}=0.3405 \times 10^{-9} \mathrm{~m}$, $m_{A}=6.6337 \times 10^{-26} \mathrm{~kg}$, and $\epsilon_{A B} / \epsilon_{A A}=1.5, \epsilon_{B B} / \epsilon_{A A}=0.5, \sigma_{A B} / \sigma_{A A}=0.8$, and $\sigma_{B B} / \sigma_{A A}=0.88, m_{B} / m A=1$. Densities are reported in units of $\rho_{0}=2.53 \times 10^{28} \mathrm{~m}^{-3}$ or $1.678 \mathrm{gcm}^{-3}$. The liquid-gas critical point is located roughly at $T_{c}=130 K, \rho_{c}=0.416 \rho_{0}$. (a) Diffusivities $D$ from molecular dynamics simulations (details of the simulations are as in [9]) are displayed in a 'fragility plot' for 5 densities. The reference temperature $T_{r}$ is chosen such that $D\left(T_{r}\right)=2.44 \times 10^{-8} \mathrm{~cm}^{2} / \mathrm{s}$, which is slightly below the lowest $D$ values measured. The VFT extrapolation is used to locate $T_{r}$. Fit values $D_{0}$ (preexponent) are in the range of 0.87 to $2.0 \times 10^{-4} \mathrm{~cm}^{2} / \mathrm{s}$, close to the experimentally observed values. (b) Fragility index $K$ obtained (i) from VFT fits to diffusivities $D$, and from the configurational entropy obtained (ii) from thermodynamic integration ('TI'), and (iii) from analysis of the potential energy landscape ('PEL'). Dimensionless thermodynamic fragility indices are obtained by dividing $K_{A G}^{T I}, K_{A G}^{P E L}$ by $N \epsilon_{A A}$. Comparison with data in [10] shows that at the low density end, the fragility of the model liquid compares with that of considerably fragile liquids such as toluene, orthoterphenyl and salol, while at the high density end, the fragility is extremely high. Temperatures of vanishing diffusivity $T_{0}$ from VFT fits are $18.76 K, 24.98 K, 35.97 K, 47.86 K$ and $75.75 K$ for $\rho / \rho_{0}=1.1,1.15,1.2,1.25$ and 1.35 respectively.

Figure 2: Adam-Gibbs plot, configurational entropy vs. temperature. (a) The 'Adam-Gibbs' plot displaying $\log (D)$ vs. $\left(T S_{c}\right)^{-1}$. $T S_{c}$ is expressed in units of $\epsilon_{A A}$. The Adam-Gibbs expectation of a linear dependence is well satisfied at each density. (b) Configurational entropy obtained from thermodynamic integration ('TI') (lines, not labeled), and from analysis of the potential energy landscape (points, labeled as 'PEL'), plotted against temperature $T$ scaled to the Kauzmann temperature $T_{K}$ obtained from thermodynamic integration. In the TI method, the total entropy $S_{\text {total }}$ of the liquid is obtained from its total free energy $F_{\text {total }}$, from $F_{\text {total }}=U_{\text {total }}-T S_{\text {total }}$, where $U_{\text {total }}$ is the internal energy. Thermodynamic identity $P=\rho^{2}\left(\frac{\partial F_{\text {total }} / N}{\partial \rho}\right)_{T}$ where $P$ is the pressure, is used to integrate $P$ from simulations to obtain $F_{\text {total }}$ with respect to the known ideal gas free energy. The identity $U_{\text {total }}=\left(\frac{\partial F_{\text {total }} / T}{\partial 1 / T}\right)_{\rho}$ is used to integrate at fixed density $U_{\text {total }}$ from simulations to obtain $F_{\text {total }}$ at any desired $T$. Extrapolations to low $T$ beyond the range of simulations utilize the form for the potential energies $E, E=E_{0}+E_{1} T^{E_{2}}$, where $E_{2} \sim 3 / 5$ (see $[7$ ]). Basin entropies $S_{v i b}$ are obtained by averaging over typical inherent structures at each $T$, whose vibrational frequencies are obtained numerically by diagonalising the Hessian (matrix of second derivatives of the potential energy). The plot covers at each density the range of $T$ where simulations have been performed. Scaling temperatures $T_{K}^{T I}$, the Kauzmann or ideal glass transition temperature where $S_{c}=0$, are: $20.44 K, 26.83 K, 34.35 K, 42.49 K$, and $62.47 K$, for $\rho / \rho_{0}=1.1,1.15,1.2,1.25$ and 1.35 respectively. $T_{K}^{P E L}$ obtained from the PEL method are, in the same order, $20.24 K, 26.18 K, 34.74 K, 44.64 K$, and $62.35 K$.

Figure 3: Distribution of energy minima, basin entropy, and temperature dependence of inherent structure energies. (a) The configurational entropy density $\mathcal{S}_{c}(\Phi)$ plotted against inherent structure energy $\Phi$ minus the 'Kauzmann' energy $\Phi_{\text {min }}$ below which $\mathcal{S}_{c}(\Phi)=0 . \quad \Phi_{\min }$ is the lower root of $\frac{\mathcal{S}_{c}(\Phi)}{N k_{B}}=\alpha-\frac{\left(\Phi-\Phi_{o}\right)^{2}}{\sigma^{2}}=0$. For each density, data from 9 temperatures (on average) are shown (smaller symbols) which collapse onto each other, validating the harmonic approximation for calculating basin entropies (see text). Also shown are the averages of these curves (larger symbols), for whose calculation a threshold of 20 samples (out of a total of 1000) is imposed in each bin. (b) The $T$-independent component of the basin entropy $\Delta S_{v i b}(\Phi)$ vs. $\Phi-\Phi_{o}$, where $\Phi_{o}$ is the value where $\mathcal{S}_{c}(\Phi)$ is maximum. $\Delta S_{v i b}(\Phi)$ curves are approximately linear. The $T$ independence of $\Delta S_{v i b}(\Phi)$ is an artefact of the classical mechanical treatment employed here, but a quantum mechanical calculation of the basin entropy does not alter the picture fundamentally (unpublished results). (c) Scaled plot of the average IS energies sampled in simulations, which are expected to collapse on to the straight line shown at low temperatures (right side of the graph), if the assumption of a Gaussian configurational entropy density $\mathcal{S}_{c}(\Phi)$ is valid (see text). The observed data collapse validates the Gaussian form used for $\mathcal{S}_{c}(\Phi)$. Deviations at high temperatures are due to the breakdown of harmonicity of basins. Fit parameters $\alpha, \phi_{0} / N \epsilon_{A A}, \sigma / N \epsilon_{A A}$ and $\delta S \epsilon_{A A} / k_{B}$ are, $\left(\rho=1.1 \rho_{0}\right): 1.010,-6.586,0.303,-0.721$, $\left(\rho=1.15 \rho_{0}\right): \quad 0.957, \quad-6.684,0.374, \quad-0.652,\left(\rho=1.2 \rho_{0}\right): 0.933, \quad-6.683,0.489, \quad-0.500,\left(\rho=1.25 \rho_{0}\right):$ $0.927,-6.564,0.642,-0.314$, and $\left(\rho=1.35 \rho_{0}\right): 0.849,-6.088,0.873,-0.188$. 
SASTRY FIGURE 1
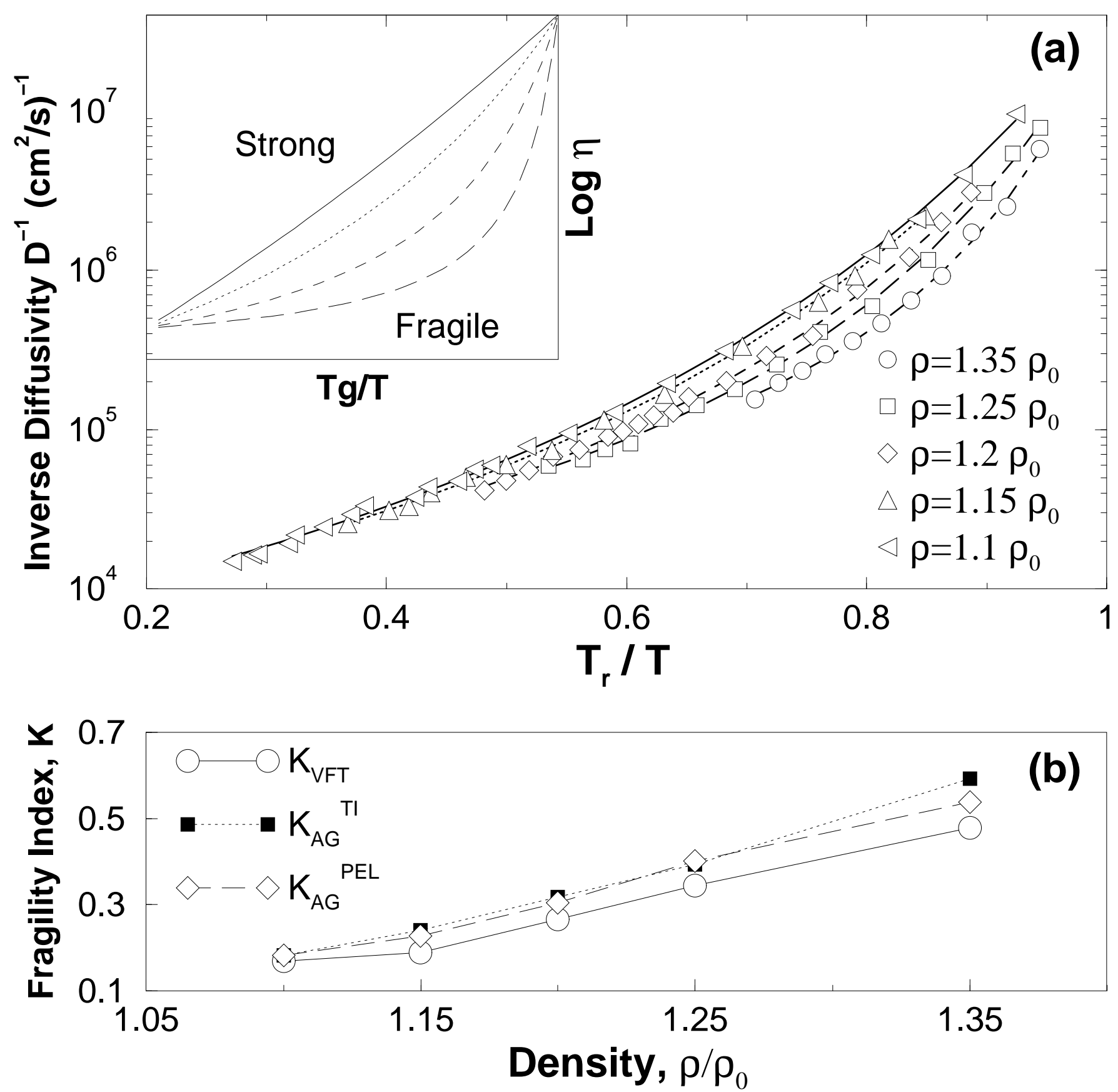
SASTRY FIGURE 2
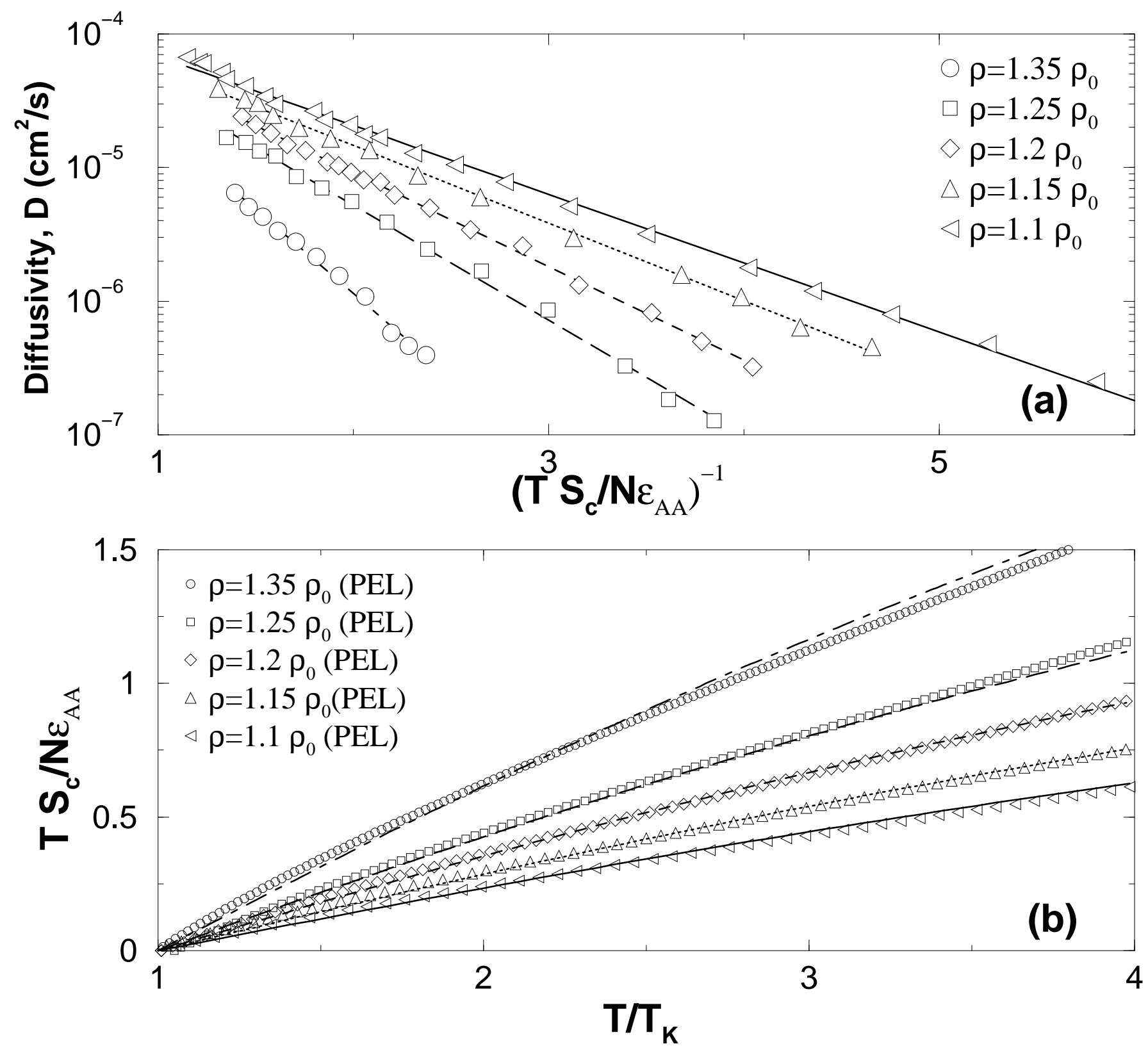
SASTRY FIGURE 3
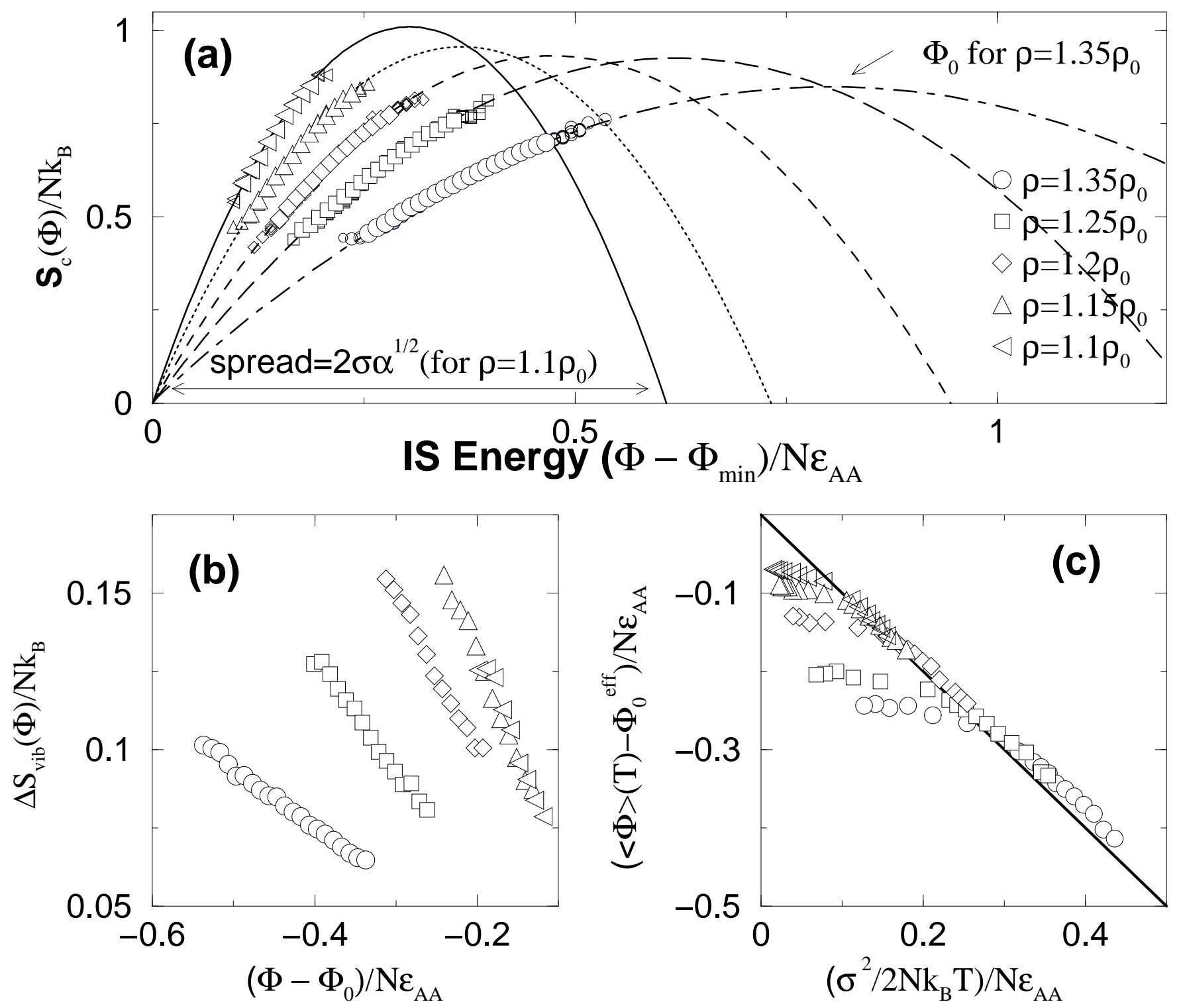